\title{
Enhancing our understanding of small bowel function using modern imaging techniques
}

\author{
Robin Spiller ${ }^{1}$ \& Caroline Hoad ${ }^{1,2}$ \\ Affiliation \\ 1 NIHR Nottingham Biomedical Research Centre \& University of Nottingham \\ 2 Sir Peter Mansfield Imaging Centre, Department of Physics, University of \\ Nottingham
}

\author{
Corresponding author \\ Professor Robin Spiller \\ Nottingham Digestive Diseases Centre \\ NIHR Nottingham Biomedical Research Centre (BRC) \\ Room WE1415 \\ Queen's Medical Centre, E floor West Block \\ Clifton Boulevard \\ Nottingham \\ NG2 7UH \\ robin.spiller@nottingham.ac.uk
}

\section{Abstract}

Small intestinal function is critical to digestive health and patients believe an abnormal reaction to food is responsible for many of their symptoms. Despite this, our ability to assess disturbed function in clinical practice has been limited, particularly after ingestion of the complex nutrients which make up normal food. Recent advances in both wireless capsules and magnetic resonance imaging have provided new insights. This review will briefly describe the limitations of past techniques and focus on how these newer techniques are changing our understanding, particularly of how patients' gastrointestinal tracts respond to food.

\section{Key words: Magnetic Resonance Imaging, small intestine, function, food, measurement}

\section{Key Points}

- Understanding of how the small intestine responds to normal mixed nutrient foods has been limited by difficulties in access

- This review outlines advances in non-invasive imaging, both endoscopic and magnetic resonance imaging which have enhanced our understanding

- There is a wide range of response to different foods which may underlie some of the food intolerances our patients report

\section{Introduction}

Our understanding of functional gastrointestinal diseases is limited by our ability to assess function and nowhere it that better illustrated than in the small bowel. Despite this being a vital organ its inaccessibility has markedly limited studies, particularly of 
how we process complex meals. When new technologies become available there are many important questions we can start to ask.

The stimulus for this review has been a burgeoning of new data derived from a range of novel patient acceptable ways of assessing function. The focus of this review will be on new methods especially MRI which have opened up the area, allowing the study of responses to complex mixed nutrient meals and in both healthy volunteers and patients.

The key functions of the small intestine are to receive acidic gastric chyme, neutralise the acid and mix it with bile to optimise the conditions for pancreatic and intestinal enzymes to allow digestion and absorption of complex nutrients.

While barium contrast radiology gives excellent definition of anatomy

it is a poor way of assessing function as the barium suspension used lacks normal nutrients.

Intestinal perfusion techniques

Assessing absorption / secretion has been performed using intestinal perfusion. This requires the passage of multi-lumen tubes into the intestine through which test solutions incorporating an non-absorbable marker, usually polyethylene glycol (PEG) are pumped and $20-30 \mathrm{~cm}$ further down the intestine the solutions are aspirated. Knowing flow and the dilution of the marker an increase or decrease in flow due to absorption or secretion can be calculated. Such studies have demonstrated the principles of absorption showing the sodium dependence of active sugar and amino acid absorption and also that water absorption is largely due to active sodium and sugar absorption with an associated passive water flux ${ }^{1}$. The requirement of aspiration up very fine tubes (3-4mm internal diameter) limits these studies to low viscosity solutions. Very few patient studies have been done, possibly because the prolonged intubation needed is uncomfortable and the tube's presence alters what is being measured, stimulating both secretion and motility 2 3 . Furthermore to facilitate aspiration unphysiological flow rates of around $20 \mathrm{ml} / \mathrm{min}$ are used which are much greater than normal flow which is around $2 \mathrm{ml} / \mathrm{min}$ in fed state 4 . Measuring motility using water perfused intestinal manometers has similar limitations to perfusion studies though the tubes are finer and better tolerated. Such studies have clearly identified abnormalities of the migrating motor complex and diseases where this is abnormal e.g. showing decreased frequency and regularity of the migrating motor complex in pseudo-obstruction due to scleroderma. However such clear results are rare and most cases of functional GI disease, especially IBS, show inconsistent abnormalities 5. Although quantitative 24 hour studies have shown some increase in frequency and amplitude of intestinal pressure waves in irritable bowel syndrome with diarrhoea (IBSD) most IBS patients have values with the normal range ${ }^{6}$ and the technique is not widely used in clinical practice.

\section{Breath tests to evaluate mucosal function and transit}

The breath tests based on the fermentation of unabsorbed carbohydrate by colonic bacteria provided a technique which was easy to apply to large numbers of patients and were initially enthusiastically adopted though recently recognition of the many limitations particularly of the lactulose and fructose breath hydrogen test including their very poor reproducibility ${ }^{7}$ means this enthusiasm has waned.

Lactose breath hydrogen test

This was the earliest breath test to be introduced to clinical practice and remains widely used though in some laboratories it has been replaced by the more convenient genetic test $^{8}$. It assesses the rise in breath hydrogen after ingesting $50 \mathrm{~g}$ of lactose which occurs when malabsorbed lactose enters the colon. This proves a good predictor of response in practice since it simulates real life e.g. milk drinking and the signal measured assessed changes in the colon which caused relevant symptoms such as flatulence and diarrhoea.

\section{Xylose breath test}

$D$-xylose is a pentameric monosaccharide which is absorbed unchanged by an active sodium dependent transporter in the upper small bowel mucosa. Around $50 \%$ of absorbed $\mathrm{D}$-xylose is rapidly excreted in the urine. Absorption is limited allowing its 
urinary excretion in the 5 hours following ingestion of $25 \mathrm{~g}$ to be used as a measure of small bowel function ${ }^{9}$. Excretion of $>5 \mathrm{~g}$ is normal while subjects with small bowel disease such as celiac disease excrete around 1-2 g. Collecting urine is inconvenient and often incomplete, so a serum xylose at 1 hour is often used and a level $<25 \mathrm{mg} / \mathrm{dl}$ considered diagnostic of malabsorption with high sensitivity and specificity ( $91 \%$ and $98 \%$ respectively) ${ }^{9}$. These tests of intestinal function were developed at a time when obtaining duodenal biopsies required intubation under radiological screening and are rarely performed now that more specific diagnoses of the causes of malabsorption can so readily be made by endoscopic duodenal biopsies.

Lactulose breath test to measure transit

Lactulose, being a disaccharide which cannot be digested by any human produces a steep rise in breath hydrogen on entering the colon, the timing of which has been used as a measure of small bowel transit. It depends on the rapid production of hydrogen (6-8 min after direct infusion into caecum via orocaecal tube ${ }^{10}$ ) which is true in most individuals, though a minority don't produce detectable hydrogen which is consumed via alternative pathways to yield methane, hydrogen sulphide or acetate. The quantitative response differs between individuals since it depends on their unique microbiota. These in turn depend on diet as seen by the fact that prefeeding with lactulose has been shown to increase the enzymes needed to ferment lactulose (b-galactosidase) ${ }^{11}$. One of its limitations is that it alters what is being assessed, since like lactose in a lactose malabsorbing patient, it induces water secretion and distension of the small bowel ${ }^{12}$ with secondary acceleration of transit ${ }^{13}$.

\section{Scintigraphic assessment of small bowel transit}

Incorporating radio-isotope into a test meal allows assessment of when isotope moves from the confines of the terminal ileum into the larger volume of the caecum and ascending colon, though this is inevitably some what subjective. This subjectivity can be reduced by measuring the $\%$ of the dose that has entered the colon at intervals and from extrapolating the plotted curves the time for $50 \%$ of isotope to enter can be estimated. The difference from the time to $50 \%$ gastric emptying then gives a measure of small bowel transit. Unlike lactulose the isotope has no effect on transit and the method does not depend on the microbiota. Its has been used to show delayed small bowel transit in chronic intestinal pseudo-obstruction ${ }^{14}$ and the acceleration of small bowel transit by bran ${ }^{15}$. While this has been used to assess drug effects ${ }^{13 ; 16-19}$ it has not been widely adopted clinically since isolated disorders of small bowel transit are uncommon ${ }^{18}$ and to some extent has been replaced by wireless capsule which avoids exposure to ionising radiation.

\section{Assessment of abnormalities of microbiota in small intestine}

The earliest clinical studies used jejunal aspirates to look for faecal organisms and concluded that these were usually associated with blind loops including jejunal diverticulae and surgically created "blind loops" which allowed colonic conditions to develop ${ }^{20}$. Subsequent studies showed that similar abnormal growth of organisms capable of deconjugating bile salts as assessed by the ${ }^{14} \mathrm{CO}_{2}$ glycocholate breath test could also be detected in patients with impaired motility and absence of the migrating motor complex ${ }^{21}$. Numerous studies make it clear that normal commensals of the oropharyngeal cavity including members of the Streptococcus, Staphylococcus Lactobacillus, Neisseria, Veillonella and Corynebacterium families will be detected in the small bowel if qastric acid is absent due to proton pump therapy, gastric atropy or other diseases such as cirrhosis. Prospective studies suggest that prolonged PPI therapy can increase SIBO and is associated with vague abdominal symptoms such as bloating and flatulence ${ }^{22}$. The presence of fecal microbiota including coliforms and Bacteroides which require a microaerophilic environment may indicate more severe abnormalities and also be associated with evidence of damage to the mucosa and frank malabsorption.

${ }^{14} \mathrm{C}$-labelled xylose was also used as a non-invasive method to show small intestinal bacteria overgrowth (SIBO) and performed well compared to jejunal aspiration ${ }^{23}$. 
However ${ }^{14} \mathrm{C}$ has a half life of 100,000 years and is retained within the body so the radiation exposure is unacceptable and these tests are no longer used though ${ }^{13} \mathrm{C}-$ xylose test is available. Intubation is impracticable in normal clinical practice and most centres will now use duodenal aspirate taken at endoscopy. At the Mayo clinic the criteria for abnormality are $>10^{5}$ colony forming units(cfu) $/ \mathrm{ml}$ or counts of anaerobic organisms of $>10^{4} \mathrm{cfu} / \mathrm{ml}$, the lower threshold recognising that anaerobic organisms represent a more abnormal state and are more likely to be associated with disease ${ }^{24}$.

Lactulose breath hydrogen test to detect small intestinal bacterial overgrowth

Quantitative culture of intestinal aspirates requires samples to be transported anerobically and rapidly processed which is very demanding in terms of time and effort so simpler alternatives would be attractive.

Early reports suggested that small intestinal bacterial overgrowth (SIBO) could be detected by the presence of an abnormal lactulose breath hydrogen test (LBHTs). IBS patients were reported as more likely to show an abnormal LBHT with either 1) a rise in breath hydrogen $<90$ minutes after ingesting $10 \mathrm{~g}$ of lactulose in $240 \mathrm{ml}$ of water or 2) a rise $>20 \mathrm{ppm}$ within 180 minutes or 3 ) the presence of two distinct peaks. However subsequent larger studies failed to show any difference between healthy controls and IBS patients using such criteria 25 26;27. A recent improvement in the lactulose breath hydrogen test incorporating a scintigraphic marker of caecal arrival of the lactulose suggested that most case of early rise in breath hydrogen were due to fast orocaecal transit ${ }^{28}$. The proportion of IBS who show a breath hydrogen rise before caecal arrival depends on the thresholds used. Using a $20 \mathrm{ppm}$ threshold suggests only $3 \%$ of IBS and no healthy controls have SIBO but this rises to $17 \%$ of patients and no controls if $10 \mathrm{ppm}$ is used ${ }^{29}$. When the more demanding criteria of jejunal aspirates having $>10^{5}$ organisms $/ \mathrm{ml}$ is used only $4 \%$ of IBS have small intestinal bacterial overgrowth ${ }^{25}$. If less stringent criteria of $>5 \times 10^{3} / \mathrm{ml}$ are used then $12 \%$ of controls and $43 \%$ of IBS patients meet criteria though this may reflect greater proton pump utilisation in IBS which is associated with similar increases in small bowel organisms. Validation against jejunal aspirate has only rarely been performed but suggests the LBHT performs poorly, with low sensitivity and specificity ${ }^{30}$.

The small benefit seen with rifaximin treatment cannot be used as evidence of the importance of SIBO since it may reflect changes in the colon rather than small bowel so the role of breath testing for SIBO in IBS remains unclear ${ }^{31}$. More direct studies on microbiota currently being done using small bowel mucosal biopsies for culture and DNAbased assessments may answer these potentially important questions.

Glucose breath hydrogen test

Measuring the breath hydrogen response after ingesting $50 \mathrm{~g}$ of glucose has been widely used and is more sensitive in detecting small intestinal bacterial overgrowth than the ${ }^{14} \mathrm{C}$-labelled xylose breath test ${ }^{32}$. However the performance of these tests requires organisms which ferment glucose to hydrogen and depends on the precise clinical groups studied. Direct comparison with aspirates suggests glucose breath hydrogen test has poor sensitivity, as low as $27 \%$ in some series ${ }^{30}$. Further more recent studies show poor correlation between bacterial numbers either by culture or direct DNA-based assessment and breath hydrogen ${ }^{33}$. Fast transit to the colon as shown by simultaneous scintigraphy is a common cause of false positives after gastric surgery ${ }^{34}$.

\section{Assessing gas in the small bowel}

Few imaging studies have examined this owing to the overlap of small bowel and colon making it difficult to precisely allocate gas bubbles to gut regions. One study using CT scanning showed that in healthy subjects the gut contained a median(interquartile range) of $95(71-141) \mathrm{ml}$ of which $68(58-83) \%$ was in the colon leaving about $1 / 3^{\text {rd }}$ in the small bowel, mostly in small bubbles containing $<6 \mathrm{ml}$. Around $5 \%$ of the total gas lay in the region of the terminal ileum, which is where bacterial fermentation of poorly absorbed dietary residue is most likely given that the terminal ileum contains around $10^{7}$ organisms per $\mathrm{ml}$. How important this is remains unclear since there was no difference in small bowel gas in patients with FGIDs compared to healthy controls ${ }^{35}$. 
A novel gas sensing radio-pill, $25 \mathrm{~mm}$ long and $9.8 \mathrm{~mm}$ in diameter which can detect methane, carbon dioxide, hydrogen and oxygen has recently been developed and used to study fermentation of carbohydrate in the gut ${ }^{36}$. The oxygen signal falls rapidly after leaving the stomach and its arrival in the anaerobic colon is readily detected so in theory it should be able to detect fermentation in the small bowel suggestive of SIBO. Results in large numbers of IBS patients are awaited with interest.

\section{Wireless capsule telemetry}

The idea of using pressure and $\mathrm{pH}$ sensitive pills which could be ingested and pass throughout the gut is not new, being first developed in the $1960 \mathrm{~s}^{37}$. However improvements in electronics have permitted the production of much improved devices allowing both endoscopy as well as assessment of pressure, $\mathrm{pH}$ and temperature ${ }^{38 ; 39}$. The SmartPill capsule measures $26 \mathrm{~mm} \times 13 \mathrm{~mm}$ making it relatively easy to swallow however its size means that it is likely to require a migrating motor complex to push it through the pylorus. This passage can be easily detected by the marked change in $\mathrm{pH}$, being around 1-2 in the fasted stomach rising to 5-6 on entering the duodenum. The $\mathrm{pH}$ thereafter rises steadily reaching around 7-8, driven by active $\mathrm{H}^{+} / \mathrm{HCO}_{3}-$ exchange in the terminal ileum. The entry into the colon is signalled by the fall in $\mathrm{pH}$ to around 5-6 in the ascending colon which contains up to $60 \mathrm{mEq} / \mathrm{l}$ of short chain fatty acids. This allows assessment of small bowel transit but it main application has been to assess colonic transit and motility in constipation. When applied to patients with constipation or nausea the finding of delayed small bowel transit may be useful in indicating a more generalised disorder suggestive of panenteric dysmotility ${ }^{40}$.

\section{Endoluminal image analysis to assess small bowel motility}

It is a major problem that intubation to perform manometry is stressful and that this undoubtedly alters both gastric and small bowel motility. A novel approach, which largely avoids this problem, has been to use capsule endoscopy to non-invasively quantify wall movements. A study of patients with manometrically diagnosed pseudo-obstruction compared with health controls showed the patients had less phasic luminal closures and more images showing no movement. It also identified a group of symptomatic patients who did not show manometric abnormalities yet did have abnormalities identified with the new technique ${ }^{41}$. Scoring the many thousands of images is very time consuming but a subsequent much larger series of 80 FGID patients using sophisticated automated image analysis showed that the method could identify around $29 \%$ with abnormalities around half having hypodynamic and the other half hyperdynamic motility ${ }^{42}$.

\section{Magnetic tracking System}

Magnet Tracking System(MTS-1) is a novel technique involving tracking the movement through the gastrointestinal tract of a small magnetic pill (dimensions: $6 \times 15 \mathrm{~mm}$, weight: $0.9 \mathrm{~g}$, density: $1.8 \mathrm{~g} \mathrm{~cm}-3)^{43}$. Its movement from stomach to small intestine can be determined from the cessation of regular $3 /$ minute movements due to gastric contractions. Ileocecal passage, which was identified as cessation of the 8-10 min-1 contraction frequency of the small intestine is more problematic, as in the ileum contractions are fewer and more irregular. The most unexpected finding using this technique is that more than half the distance covered during the first two hours in the small intestine is due to fast movements which only occupy $5 \%$ of the time. This was true both fasting and fed ${ }^{44}$. The MMC may well account for rapid fasting movements but the large rapid movements during feeding are unexpected. However these may be important in spreading chime along the small bowel to speed up absorption. Exactly the role of this technique in clinical practice or research remains unclear, however measuring transit alone is of limited value and usually needs to be combined with assessments of other small bowel functions such as absorption / secretion.

\section{Magnetic Resonance Imaging of small bowel water}

Clinical use

Modern imaging techniques, which allow high quality images to be obtained within a few hundred milliseconds, together with the lack of ionising radiation have made MRI a very attractive way of assessing small bowel anatomy in clinical practice. This is particularly 
true for patients with Crohn's disease, who often require repeated imaging over a life time, which in the past has exposed them to potential harmful doses of radiation. However the use of large doses of osmotic agents such as mannitol, while providing excellent anatomical definition of mucosal pathology does disturb digestive function and a rather different technique using the undisturbed bowel is required to assess function.

Measuring small bowel water content

The technique of MRI utilises the high concentration of hydrogen protons in the body and their magnetic properties when placed in a powerful magnetic field. The protons are excited by a radiofrequency excitation pulse and this energy is then re-emitted and analysed. Since the frequencies emitted depend on the magnetic field strength, spatial information can be encoded using time varying magnetic field gradients. Different contrast in the images can be acquired using different timings and combinations of the radiofrequency electromagnetic waves used to excite the protons with the magnetic field gradients used to encode positional information. This is because the chemical and physical environment of the protons effects how fast the signal decays and returns to equilibrium. This decay and return to equilibrium of the MRI signal are characterised by the relaxation parameters T1 (return to equilibrium) and T2 (signal decay).

The liquid in the small bowel can be assessed with a heavily T2-weighted single shot, fast spin echo MRI sequence (similar to that used for magnetic resonance cholangiopancreatography [MRCP])to acquire 24 coronal images, with in-plane resolution interpolated to $0.78 \mathrm{~mm} \times 0.78 \mathrm{~mm}$ and a slice thickness of $7 \mathrm{~mm}$ in a single breathhold. This sequence yields high-intensity signals from areas with non-viscous ('free') fluid and little signal from body tissues ${ }^{45}$ (Figure $1 \mathrm{~A}$ ). This sequence and analysis method to measure the volume of small bowel 'free' water has been validated using an infusion of known amounts of a mannitol-saline solution, known from perfusion studies to show no net absorption or secretion ${ }^{46}$. The mannitol-saline solution was instilled (via a nasoduodenal tube) in boluses of $40 \mathrm{ml}$ up to a total of $320 \mathrm{ml}$ and the resulting images used to assess the volume. The exclusion of other (non fluid) tissue was achieved by using the signal intensity from the cerebrospinal fluid to calculate a cut off, excluding all pixels with values below this threshold. The clearly identifiable gallbladder, kidneys, bladder, stomach and large mesenteric vessels were then excluded manually. The threshold was optimised to give the best correlation of measured with infused volume and this was then validated on a second set of data (Figure 1B).

This validated method has subsequently been used to define the changes in small bowel volume during digestion of a range of different components of food.

\section{Osmotic effect of poorly absorbed solutes}

Lactose intolerance has been well recognised for over 50 years as a trigger of IBS like symptoms. Intubation studies using intestinal aspirates obtained after ingestion of $50 \mathrm{~g}$ lactose in $180 \mathrm{ml}$ of water showed considerable dilution of non-absorbable marker, indicating that approximately $1600 \mathrm{ml}$ of water entered the small bowel, driven by the low sodium and hypertonicity of the ingested solution ${ }^{47}$. A similar secretion has been documented using MRI. Studies comparing an iso-osmolar mannitol solution $(17.5 \mathrm{~g}$ in $350 \mathrm{ml}$ water) with an iso-osmolar glucose solution $(17.5 \mathrm{~g}$ glucose in $350 \mathrm{ml}$ water showed both solutions emptied rapidly from the stomach and 40 minutes after ingestion $250 \mathrm{ml}$ of the glucose and $276 \mathrm{ml}$ of the mannitol solution had emptied into the small bowel. While the glucose solution was rapidly absorbed after mannitol it peaked at over 4 times baseline at 40 minutes (Figure 2) ${ }^{48}$. This peak is $50 \mathrm{ml}$ higher than the sum of the volume emptied from the stomach plus the baseline value, indicating a net secretion induced by the mannitol. This confirms previous perfusion studies but without the need for intubation, making such studies much easier to perform. The secretory effect of mannitol was exploited as bowel preparation for colonoscopy before this was superseded by the even more effective polyethylene glycol and electrolyte mixtures now used. MRI studies showed that Moviprep, a PEG solution containing sodium chloride, sulphate and ascorbate caused a rapid increase in SBWC, from a baseline of $80-100 \mathrm{ml}$ rising to peak around $650 \mathrm{ml}$ after $1 \mathrm{I}$ of Moviprep. ${ }^{49}$ (Figure 3). There was also a rapid transfer of fluid to the colon which showed as a much brighter image with an associated rise in T2. The 
effect of mannitol on the colon was much less apparent and, at the dose used, the colonic images changed surprisingly little, indicating that most of the mannitol was fermented and the resulting SCFAs absorbed along with water.

Assessment of digestion of complex foods

Most food we eat is not composed of simple solutions but complex mixtures of liquid and solid. Sampling these using intestinal tubes is virtually impossible but MRI allows a noninvasive assessment of fluid fluxes, intestinal distension and entry of meal residue into the colon with subsequent fermentation, all features which can be linked to a range of symptoms described by patients with FGIDs.

Carbohydrate, derived from polymers used by plants to store energy such as wheat, rice, potatoes and other tubers, is a dominant part of most patient's diets. Cooked rice is a particularly well tolerated food, being rapidly and fairly completely absorbed as shown by breath hydrogen testing ${ }^{50}$. We have used $220 \mathrm{~g}$ cooked rice as part of a mixed nutrient standard test meal combined with a $100 \mathrm{ml}$ orange juice, jam and bran flakes providing $362 \mathrm{kcal}$. After ingestion the changes in SBWC are biphasic. The gastric images show that the liquid phase empties faster than the solid. The liquid contains glucose, fructose and sucrose which are rapidly absorbed causing SBWC to fall to a nadir at 90 minutes post ingestion (Figure 4). The SBWC then rises in the later phase when the rice and bran enter the small bowel. The two lines show the meal with and without the bran, which increased SBWC. Bran also shortened transit from duodenum to caecum, implying an increased flow due to stimulation of secretion ${ }^{48}$. This effect was more marked in females than males, though why this should be so is unclear. An earlier scintigraphic study using an identical protocol had shown similarly accelerated transit ${ }^{15}$ but unlike the MRI study could make no inferences about the cause, which demonstrates the power of the new technique compared with the old one.

\section{Gastrointestinal response to FODMAPs}

The recognition that poorly absorbed but rapidly fermentable dietary carbohydrate, so called FOMAPs (fermentable oligo-di- and mono-saccharide and polyhydric alcohols) was responsible for at least some of the symptoms of the ubiquitous irritable bowel syndrome ${ }^{51}$ has led to a substantial improvement in the management of IBS. Understanding exactly how FODMAPs cause symptoms requires imaging after a provocative meal containing FODMAPs. MRI studies showed clearly that, while the poorly absorbed but osmotically active monomer, fructose, distended the small bowel with fluid, this was not seen with the larger less osmotically active molecule inulin. This passes through the small bowel without causing distension but is rapidly fermented once it enters the ascending colon, producing obvious gaseous distension ${ }^{52}$. Interestingly IBS patients did not appear to show any greater physiological response to either fructose or inulin compared to healthy volunteers but had markedly greater symptoms, reflecting their known visceral hypersensitivity ${ }^{53}$. The best correlation with patients' composite symptom score (CSS) was with peak increase in colonic gas volume after inulin. A weaker correlation between SBWC and symptoms was seen after fructose in keeping with its greater impact in the small bowel.

Many patients believe that bread causes symptoms particularly bloating and a low FODMAP diet often involves excluding normal bread which contains about $1 \%$ FODMAPs, mostly fructo-oligosaccharides including raffinose and stachyose ${ }^{54}$. While this might lead to excess colonic gas and hence bloating / gas 4-6 hours after ingestion, the bloating developing immediately after eating is more likely due to gastric distension. MRI studies comparing equicaloric rice meal with whole meal bread showed that while the rice pudding meal separated into a solid and liquid phase, the whole meal bread formed a rather homogeneous mass with no clear separation of fluid and solid. While the rice meal allowed the stomach to sieve the meal and empty the fluid faster than the solid, sieving was not possible with the more homogeneous bread meal and as a consequence the gastric volume fell more slowly. In contrast once the wheat entered the small intestine it was rapidly absorbed and the SBWC was consistently lower than after the rice meal postprandially. This suggests than any sensation of bloating at this time was more likely to come from the stomach than the small intestine ${ }^{55}$. 
Gluten is a key determinant of dough elasticity and thought to contribute to symptoms so we have investigated how it affects the upper intestinal processing of bread meals by comparing gluten free bread with bread with normal gluten content and a bread supplemented with 3\% gluten. The gastric images showed the added gluten did produce a less homogeneous intra-gastric appearance but this was not associated with any difference in gastric emptying and all meals showed the same pronounced drop in small bowel water postprandially ${ }^{56}$. Unexpectedly feeding gluten free bread for 2 days significantly increased the fasting transverse colonic volumes by $36 \%$ compared to normal bread, possibly reflecting the effect of the tapioca and potato starches used to replace wheat in gluten free products.

\section{Impact of dietary fibre on small bowel contents}

Dietary fibre, which is widely used as a dietary treatment for constipation, comprises a range of very different materials often divided into soluble and insoluble forms. Precisely how these produce laxation and the role of the small bowel in this has been unclear. Ileostomy studies suggested that soluble fibre such as psyllium and gelling agents increase small bowel flow but the small bowel of ileostomists is rather atypical with abnormal mucosa and microbiota so it has been important to study this in intact humans. It is only recently that MRI has enabled us to complete this part of the picture. Psyllium appears to trap water in the small bowel, presumably by increasing viscosity thereby impairing convection and reducing access of water to the intestinal mucosa where absorption takes place. Conventional doses of 3.5 and $7 \mathrm{~g}$ psyllium three times daily with food, lead to increased post prandial SBWC, which rises steadily during the day until a large $(1000 \mathrm{kcal})$ meal stimulates ileal emptying, reducing SBWC back towards baseline (Figure 5$)^{57}$. Our earlier studies with bran ${ }^{58}$ and more recent studies comparing bran directly with psyllium and prickly pear extract ${ }^{59}$ show similar increase in SBWC developing postprandially. The increase after bran appears to be more likely due to stimulation of secretion since unlike psyllium it shows little water trapping ${ }^{60}$. The resulting ileal contents are emptied into the ascending colon after the second large meal which stimulates ileal emptying. This leads to an increase in the fluidity of the ascending colon contents. As already stated, T1 is a time constant reflecting the decay of magnetisation after excitation by radiofrequency energy pulses and increases as the colonic contents become more watery. During the psyllium study the T1 was significantly higher after both $3.5 \mathrm{~g}$ and $7 \mathrm{~g}$ psyllium being $631 \pm 37$ and $779 \pm 48 \mathrm{msec}$ respectively compared to the maltodextrin control at $447 \pm 37 \mathrm{msec}$. We validated the use of T1 measurements as an assessment of colonic water content by showing that T1 of descending colon contents were well correlated with the \% water of stool passed subsequently, Pearson's $r=0.65^{61}$.

\section{Effect of phytochemicals}

While carbohydrate is the staple source of energy, much of what we eat is flavoured with plant products such as herbs and spices, all of which influence the processes of digestion. Rhubarb is an important ingredient of many Chinese herbal preparations and reported by ileostomists to increase ileal outflow ${ }^{62}$. Imaging the bowel after rhubarb ingestion shows a large increase in SBWC, followed by a rise in ascending colon T1 63 . Rhubarb is known to contain rhein, a mild cyotoxic substance, shown in animal studies to exert a laxative effect which can be blocked by indomethacin. Rhein stimulates prostaglandin 3 secretion and downregulates expression of Aquaporin 3 in cell lines which is a possible mechanism of its laxative effect ${ }^{64}$. Interestingly the same study also showed that lettuce which contains a milk-like latex, lactucarium, which like rhein is a mild cytotoxin also increases SBWC ${ }^{65}$. It is likely that many green vegetables similarly increase SBWC and hence delivery of fluid to the colon with likely beneficial effects in preventing constipation.

\section{Abnormalities of small bowel in coeliac disease and scleroderma}

Both coeliac disease and scleroderma have a well defined appearance in barium contrast studies but as mentioned barium is a nonphysiological stimulus. MRI can demonstrate similar anatomical abnormalities such as dilated loops (Figure 6) with the potential to 
further understanding of pathophysiology (Lam et al in press 2019). The dilated fasting loops are likely to reflect the effect of reduced migrating motor complexes and loss of their normal function of propelling meal residue into the colon. Whether increased fasting SBWC is associated with SIBO could be easily determined and would improve our understanding and management of these conditions.

Abnormalities of small bowel in IBS

One of the big surprises of recent MRI studies was the demonstration that IBS patients with diarrhoea (IBS-D) had less small bowel water than normal (Figure 6) ${ }^{48 ; 66}$. This was associated with accelerated mouth to caecum transit and anxiety ${ }^{40}$, suggesting that psychological stress might be causing increased tone, leading to decreased SBWC. Previous human studies using a dichotomous listening test as a stressor have shown that psychological stress accelerates small bowel transit ${ }^{67}$. Subsequent animal studies had suggested that this involved activation of the hypothalamic pituitary axis mediated by corticotrophin releasing hormone $(\mathrm{CRH})$. $\mathrm{CRH}$ also acts peripherally to accelerate transit ${ }^{68}$ and can be readily used in humans to mimic the effect of stress on the gut. $A$ randomised placebo controlled trial (RCT) in healthy volunteers of an intravenous bolus of $100 \mathrm{mcg} \mathrm{CRH}$ versus saline control showed that this reduced SBWC postprandially by an amount similar to that seen in IBS-D. CRH also increased the volume of the ascending colon in the following 3 hours suggesting that stress causes an increased inflow into the colon ${ }^{69}$. Using the same study design but using a fructose meal we were also able to show not only that $\mathrm{CRH}$ constricted the small bowel but that this also enhanced the subsequent increase in ascending colon volumes showing how stress could exacerbate the impact of FODMAPs 70 .

While studies in IBS with constipation (IBS-C) show normal fasting and postprandial SBWC, when stressed by a large dose of osmotic laxative, the SBWC was higher in functional constipation than in IBS-C suggesting an impairment of motility with delay in transit of the osmotic bolus to the colon in this subgroup ${ }^{71}$.

\section{Effect of therapeutic interventions}

As described above MRI can be used to demonstrate the mode of action of psyllium. It has also been used to show that Ondansetron, a 5HT-3 receptor antagonist (5-HT3RA) shown to slow whole gut transit in IBS-D ${ }^{72}$, increased fasting SBWC in intubated healthy volunteers while reducing antro-duodenal motility. This supports the idea that reduced fasting SBWC such as is seen in IBS-D could be an indicator of increased small bowel tonic or phasic contractions.

Loperamide is known to slow whole gut transit and to be effective in treating diarrhoea but the precise mode of action has not been shown in intact humans. A RCT comparing two formulations of loperamide versus placebo in mannitol-induced small bowel secretion showed its impact on the small bowel, with reduced SBWC and delayed arrival of fluid in the ascending colon suggesting it enhanced absorption ${ }^{73}$.

\section{Assessment of small bowel and colonic motility using MRI}

The high quality images of the colon and the ability to acquire images in milliseconds has made it possible to produce cine images of the colonic wall over several minutes and to thus quantify colonic motility ${ }^{71}$. A key requirement for this is the ability to allow image acquisition during free breathing by compensating for respiratory movement by means of an image registration technique. By deforming all the different images from the cine acquisition so that they best match a reference image (from the same dataset), it is possible to derive this deformation field information. This information then allows tracking of any location in the bowel through the cine data, and provides information on the local expansion and contraction of each pixel in the image over the acquisition time, with large changes in expansion and contraction associated with higher motility.

Summary metrics can then be used to quantify the amount of movement seen as a numerical comparison between different people or treatments. The technique was first used to demonstrate reduced motility in Crohn's ileitis ${ }^{74}$. The method has been validated by showing that it can detect the stimulatory and inhibitory effects of neostigmine and buscopan respectively on small bowel motility ${ }^{75}$. It has recently been used to show 
impaired small bowel motility in pseudo-obstruction and also to evaluate patients' response to prokinetic neostigmine ${ }^{76}$. The method continues to evolve and more recently motility has been used to measure the stimulatory effect of nutrients without the need to distend the bowel with mannitol with good reproducibility $77 ; 78$.

Future studies

MRI studies are highly patient acceptable and this makes it possible to perform repeated studies using RCT design, ideal for drug evaluation to provide early signal of effectiveness and mode of action. It also lends itself to mechanistic studies of the impact of different foods designed for specific function in both health and disease. We are currently developing MR suitable markers which could be used to more accurately assess small bowel transit times and hence assess flow. Future technical developments include the possibilities of exploiting the effects of $\mathrm{pH}$ and redox potential on the MR signal probing these potentially important parameters in health and disease.

Legend for Figures

Figure $1 \mathrm{MR}$ images of small bowel during infusion of a balance saline mannitol solution used to validate the measurement of small bowel water content

A) Maximum intensity projection images of small bowel after sequential boluses of $40 \mathrm{ml}$ mannitol-saline given via a nasoduodenal tube

B) Correlation between MRI measurement of small bowel water on the $y$ axis and infused volume + baseline volume during nasoduodenal infusion of boluses of mannitol-saline solution showing excellent agreement between the 2 measures $^{45}$.

Figure 2

Small bowel water content over time following ingestion of two iso-osmolar solutions of glucose $5 \%$ and mannitol $5 \%$. There was a striking rise in water content with mannitol compared to the rapid absorption of the glucose solution ${ }^{48}$.

Figure 3 Effect of $1 \mathrm{~L}$ and $2 \mathrm{~L}$ Moviprep on Small Bowel Water Content (SBWC).

A) Chart showing SBWC in $\mathrm{ml}$ at varying time points. Ingestion finished at time 0 with 1 hour allowed for $1 \mathrm{~L}$ and 2 hours for $2 \mathrm{~L}$ of Moviprep. Scans thereafter were at hourly intervals for the next 4 hours $(1 \mathrm{~L})$ or 6 hours $(2 \mathrm{~L})$. There was a rapid increase in SBWC peaking immediately after ingestion with a subsequent decline as fluid moved into the colon.

B) T2 weighted MR image showing water as bright pixels immediately after a 2 hour ingestion of $2 \mathrm{~L}$ of Moviprep. The stomach is dilated and the small bowel distended with fluid, some of which has entered the colon filling and distending the ascending and transverse colon ${ }^{49}$.

Figure 4 Effect of mixed nutrient liquid and solid meal on small bowel water content (SBWC)

Time profile of SBWC in $\mathrm{ml}$ against time after ingestion of orange juice and rice pudding mixed with jam with and without $15 \mathrm{~g}$ bran flakes ${ }^{48}$. Bran increased fluid in the second half of the study.

Figure 5 Effect of psyllium on small bowel water content

Small bowel water content during the study day during a RCT of psyllium $3.5 \mathrm{~g}$ and $7 \mathrm{~g}$ versus maltodextrin $14 \mathrm{~g}$ three time daily (shown by arrows) showing a rise in SBWC particularly after the second and third dose ${ }^{61}$.

Figure 6 MR images showing small bowel fluid in fasting subjects. Healthy controls (A) show little free water while both coeliac disease (B) and scleroderma (C) are 
characterised by dilated loops with increased small bowel water reflecting impaired fasting motility.

Figure 7 Effect of irritable bowel syndrome with diarrhoea on small bowel water content Small bowel water content fasting and after a $362 \mathrm{kcal}$ rice pudding and bran test meal showing the results from 26 IBS patients with diarrhoea ( lower line) who had consistently lower SBWC than 16 healthy volunteers (upper line).

\section{Reference List}

(1) Spiller RC, Jones BJ, Silk DB. Jejunal water and electrolyte absorption from two proprietary enteral feeds in man: importance of sodium content. Gut 1987; 28(6):681-687.

(2) Read NW, Al Janabi MN, Bates TE, Barber DC. Effect of gastrointestinal intubation on the passage of a solid meal through the stomach and small intestine in humans. Gastroenterology 1983; 84(6):1568-1572.

(3) Marciani L, Wright J, Foley S, Hoad CL, Totman JJ, Bush D et al. Effects of a 5$\mathrm{HT}(3)$ antagonist, ondansetron, on fasting and postprandial small bowel water content assessed by magnetic resonance imaging. Aliment Pharmacol Ther 2010; 32(5):655-663.

(4) Ladas SD, Isaacs PE, Murphy GM, Sladen GE. Fasting and postprandial ileal function in adapted ileostomates and normal subjects. Gut 1986; 27(8):906-912.

(5) Kellow JE, Phillips SF. Altered small bowel motility in irritable bowel syndrome is correlated with symptoms. Gastroenterology 1987; 92(6):1885-1893.

(6) Cogliandro RF, Antonucci A, De GR, Barbara G, Cremon C, Cogliandro L et al. Patient-reported outcomes and gut dysmotility in functional gastrointestinal disorders. Neurogastroenterol Motil 2011; 23(12):1084-1091.

(7) Yao CK, Tuck CJ, Barrett JS, Canale KE, Philpott HL, Gibson PR. Poor reproducibility of breath hydrogen testing: Implications for its application in functional bowel disorders. United European Gastroenterol J 2017; 5(2):284-292.

(8) Babu J, Kumar S, Babu P, Prasad JH, Ghoshal UC. Frequency of lactose malabsorption among healthy southern and northern Indian populations by genetic analysis and lactose hydrogen breath and tolerance tests. Am J Clin Nutr 2010; 91(1):140-146.

(9) Craig RM, Atkinson AJ Jr. D-Xylose testing: A review. Gastroenterology 1988; 95:223-231.

(10) Kellow JE, Borody TJ, Phillips SF, et al. Sulfapyridine appearance in plasma after salicylazosulfapyridine. Another simple measure of intestinal transit. Gastroenterology 1986; 91:396-400.

(11) Florent C, Flourie B, Leblond A, Rautureau M, Bernier JJ, Rambaud JC. Influence of chronic lactulose ingestion on the colonic metabolism of lactulose in man (an in vivo study). J Clin Invest 1985; 75(2):608-613.

(12) Undseth R, Berstad A, Klow NE, Arnljot K, Moi KS, Valeur J. Abnormal accumulation of intestinal fluid following ingestion of an unabsorbable 
carbohydrate in patients with irritable bowel syndrome: an MRI study.

Neurogastroenterol Motil 2014; 26(12):1686-1693.

(13) Miller MA, Parkman HP, Urbain JL, Brown KL, Donahue DJ, Knight LC et al. Comparison of scintigraphy and lactulose breath hydrogen test for assessment of orocecal transit: lactulose accelerates small bowel transit. Dig Dis Sci 1997; 42(1): $10-18$.

(14) Camilleri M, Brown ML, Malagelada JR. Impaired transit of chyme in chronic intestinal pseudoobstruction. Correction by cisapride. Gastroenterology 1986; 91(3):619-626.

(15) McIntyre A, Vincent RM, Perkins AC, Spiller RC. Effect of bran, ispaghula, and inert plastic particles on gastric emptying and small bowel transit in humans: the role of physical factors. Gut 1997; 40(2):223-227.

(16) Coleman NS, Marciani L, Blackshaw E, Wright J, Parker M, Yano T et al. Effect of a novel 5-HT3 receptor agonist MKC-733 on upper gastrointestinal motility in humans. Aliment Pharmacol Ther 2003; 18(10):1039-1048.

(17) Prather CM, Camilleri M, Zinsmeister AR, McKinzie S, Thomforde G. Tegaserod accelerates orocecal transit in patients with constipation-predominant irritable bowel syndrome. Gastroenterology 2000; 118(3):463-468.

(18) Bonapace ES, Maurer AH, Davidoff S, Krevsky B, Fisher RS, Parkman HP. Whole gut transit scintigraphy in the clinical evaluation of patients with upper and lower gastrointestinal symptoms. Am J Gastroenterol 2000; 95(10):2838-2847.

(19) Bouras EP, Camilleri M, Burton DD, Thomforde G, McKinzie S, Zinsmeister AR. Prucalopride accelerates gastrointestinal and colonic transit in patients with constipation without a rectal evacuation disorder. Gastroenterology 2001; 120(2):354-360.

(20) Drasar BS, Shiner M. Studies on the intestinal flora. II. Bacterial flora of the small intestine in patients with gastrointestinal disorders. Gut 1969; 10(10):812-819.

(21) Vantrappen G, Janssens J, Hellemans J, Ghoos Y. The interdigestive motor complex of normal subjects and patients with bacterial overgrowth of the small intestine. J Clin Invest 1977; 59(6):1158-1166.

(22) Compare D, Pica L, Rocco A, De GF, Cuomo R, Sarnelli G et al. Effects of longterm PPI treatment on producing bowel symptoms and SIBO. Eur J Clin Invest $2011 ; 41(4): 380-386$.

(23) Rumessen JJ, Gudmand-Hoyer E, Bachmann E, Justesen T. Diagnosis of bacterial overgrowth of the small intestine. Comparison of the 14C-D-xylose breath test and jejunal cultures in 60 patients. Scand J Gastroenterol 1985; 20(10):12671275.

(24) Choung RS, Ruff KC, Malhotra A, Herrick L, Locke GR, III, Harmsen WS et al. Clinical predictors of small intestinal bacterial overgrowth by duodenal aspirate culture. Aliment Pharmacol Ther 2011; 33(9):1059-1067.

(25) Posserud I, Stotzer PO, Bjornsson ES, Abrahamsson H, Simren M. Small intestinal bacterial overgrowth in patients with irritable bowel syndrome. Gut 2007; 56(6):802-808. 
(26) Walters B, Vanner SJ. Detection of bacterial overgrowth in IBS using the lactulose $\mathrm{H} 2$ breath test: comparison with 14C-D-xylose and healthy controls. Am J Gastroenterol 2005; 100(7):1566-1570.

(27) Bratten JR, Spanier J, Jones MP. Lactulose breath testing does not discriminate patients with irritable bowel syndrome from healthy controls. Am J Gastroenterol $2008 ; 103(4): 958-963$.

(28) Yu D, Cheeseman F, Vanner S. Combined oro-caecal scintigraphy and lactulose hydrogen breath testing demonstrate that breath testing detects oro-caecal transit, not small intestinal bacterial overgrowth in patients with IBS. Gut 2011; $60(3): 334-340$.

(29) Zhao J, Zheng $X$, Chu $\mathrm{H}$, Zhao J, Cong $\mathrm{Y}$, Fried $\mathrm{M}$ et al. A study of the methodological and clinical validity of the combined lactulose hydrogen breath test with scintigraphic oro-cecal transit test for diagnosing small intestinal bacterial overgrowth in IBS patients. Neurogastroenterol Motil 2014; 26(6):794802.

(30) Ghoshal UC, Srivastava D, Ghoshal U, Misra A. Breath tests in the diagnosis of small intestinal bacterial overgrowth in patients with irritable bowel syndrome in comparison with quantitative upper gut aspirate culture. Eur J Gastroenterol Hepatol 2014; 26(7):753-760.

(31) Ford AC, Spiegel BM, Talley NJ, Moayyedi P. Small intestinal bacterial overgrowth in irritable bowel syndrome: systematic review and meta-analysis. Clin Gastroenterol Hepatol 2009; 7(12):1279-1286.

(32) Stotzer PO, Kilander AF. Comparison of the 1-gram (14)C-D-xylose breath test and the 50-gram hydrogen glucose breath test for diagnosis of small intestinal bacterial overgrowth. Digestion 2000; 61(3):165-171.

(33) Sundin $\mathrm{OH}$, Mendoza-Ladd A, Morales E, Fagan BM, Zeng M, Diaz-Arevalo D et al. Does a glucose-based hydrogen and methane breath test detect bacterial overgrowth in the jejunum? Neurogastroenterol Motil 2018; 30(11):e13350.

(34) Lin EC, Massey BT. Scintigraphy Demonstrates High Rate of False-positive Results From Glucose Breath Tests for Small Bowel Bacterial Overgrowth. Clin Gastroenterol Hepatol 2016; 14(2):203-208.

(35) Bendezu RA, Barba E, Burri E, Cisternas D, Malagelada C, Segui S et al. Intestinal gas content and distribution in health and in patients with functional gut symptoms. Neurogastroenterol Motil 2015; 27(9):1249-1257.

(36) Berean KJ, Ha N, Ou JZ, Chrimes AF, Grando D, Yao CK et al. The safety and sensitivity of a telemetric capsule to monitor gastrointestinal hydrogen production in vivo in healthy subjects: a pilot trial comparison to concurrent breath analysis. Aliment Pharmacol Ther 2018; 48(6):646-654.

(37) Connell AM, ROWLANDS EN. Wireless telemetering from the digestive tract. Gut $1960 ; 1: 266-272$.

(38) Camilleri M, Thorne NK, Ringel Y, Hasler WL, Kuo B, Esfandyari T et al. Wireless $\mathrm{pH}$-motility capsule for colonic transit: prospective comparison with radiopaque markers in chronic constipation. Neurogastroenterol Motil 2010; 22(8):874-82, e233. 
(39) Rao SS, Kuo B, McCallum RW, Chey WD, DiBaise JK, Hasler WL et al. Investigation of colonic and whole-gut transit with wireless motility capsule and radiopaque markers in constipation. Clin Gastroenterol Hepatol 2009; 7(5):537544.

(40) Rao SS, Mysore K, Attaluri A, Valestin J. Diagnostic utility of wireless motility capsule in gastrointestinal dysmotility. J Clin Gastroenterol 2011; 45(8):684-690.

(41) Malagelada C, de IF, Azpiroz F, Accarino A, Segui S, Radeva P et al. New insight into intestinal motor function via noninvasive endoluminal image analysis. Gastroenterology 2008; 135(4):1155-1162.

(42) Malagelada C, Drozdzal M, Segui S, Mendez S, Vitria J, Radeva P et al. Classification of functional bowel disorders by objective physiological criteria based on endoluminal image analysis. Am J Physiol Gastrointest Liver Physiol 2015; 309(6):G413-G419.

(43) Stathopoulos E, Schlageter V, Meyrat B, Ribaupierre Y, Kucera P. Magnetic pill tracking: a novel non-invasive tool for investigation of human digestive motility. Neurogastroenterol Motil 2005; 17(1):148-154.

(44) Worsoe J, Fynne L, Gregersen T, Schlageter V, Christensen LA, Dahlerup JF et al. Gastric transit and small intestinal transit time and motility assessed by a magnet tracking system. BMC Gastroenterol 2011; 11:145.

(45) Hoad CL, Marciani L, Foley S, Totman JJ, Wright J, Bush D et al. Non-invasive quantification of small bowel water content by MRI: a validation study. Phys Med Biol 2007; 52(23):6909-6922.

(46) Spiller RC, Higgins BE, Frost PG, Silk DB. Inhibition of jejunal water and electrolyte absorption by therapeutic doses of clindamycin in man. Clin Sci (Lond) $1984 ; 67(1): 117-120$.

(47) Christopher NL, Bayless TM. Role of the small bowel and colon in lactose-induced diarrhea. Gastroenterology 1971; 60(5):845-852.

(48) Marciani L, Cox EF, Hoad CL, Pritchard S, Totman JJ, Foley S et al. Postprandial changes in small bowel water content in healthy subjects and patients with irritable bowel syndrome. Gastroenterology 2010; 138(2):469-77, 477.

(49) Marciani L, Garsed KC, Hoad CL, Fields A, Fordham I, Pritchard SE et al. Stimulation of colonic motility by oral PEG electrolyte bowel preparation assessed by MRI: comparison of split vs single dose. Neurogastroenterol Motil 2014; 26(10): 1426-1436.

(50) Kerlin P, Wong L, Harris B, Capra S. Rice flour, breath hydrogen, and malabsorption. Gastroenterology 1984; 87(3):578-585.

(51) Shepherd SJ, Parker FC, Muir JG, Gibson PR. Dietary triggers of abdominal symptoms in patients with irritable bowel syndrome: randomized placebocontrolled evidence. Clin Gastroenterol Hepatol 2008; 6(7):765-771.

(52) Murray K, Wilkinson-Smith V, Hoad C, Costigan C, Cox E, Lam C et al. Differential effects of FODMAPs (fermentable oligo-, di-, mono-saccharides and polyols) on small and large intestinal contents in healthy subjects shown by MRI. Am J Gastroenterol 2014; 109(1):110-119. 
(53) Major G, Pritchard S, Murray K, Alappadan JP, Hoad CL, Marciani L et al. Colon Hypersensitivity to Distension, Rather Than Excessive Gas Production, Produces Carbohydrate-Related Symptoms in Individuals With Irritable Bowel Syndrome. Gastroenterology 2017; 152(1):124-133.

(54) Biesiekierski JR, Rosella O, Rose R, Liels K, Barrett JS, Shepherd SJ et al. Quantification of fructans, galacto-oligosacharides and other short-chain carbohydrates in processed grains and cereals. J Hum Nutr Diet 2011; 24(2):154176.

(55) Marciani L, Pritchard SE, Hellier-Woods C, Costigan C, Hoad CL, Gowland PA et al. Delayed gastric emptying and reduced postprandial small bowel water content of equicaloric whole meal bread versus rice meals in healthy subjects: novel MRI insights. Eur J Clin Nutr 2013; 67(7):754-758.

(56) Coletta M, Gates FK, Marciani L, Shiwani H, Major G, Hoad CL et al. Effect of bread gluten content on gastrointestinal function: a crossover MRI study on healthy humans. Br J Nutr 2016; 115(1):55-61.

(57) Major G, Murray K, Singh G, Nowak A, Hoad CL, Marciani L et al. Demonstration of differences in colonic volumes, transit, chyme consistency, and response to psyllium between healthy and constipated subjects using magnetic resonance imaging. Neurogastroenterol Motil 2018; 30(9):e13400.

(58) Marciani L, Cox EF, Hoad CL, Pritchard S, Totman JJ, Foley S et al. Postprandial changes in small bowel water content in healthy subjects and patients with irritable bowel syndrome. Gastroenterology 2010; 138(2):469-77, 477.

(59) Gunn D, Murthy R, Major G, Wilkinson-Smith V, Hoad C, Gill G et al. Varying effects of bran, psyllium and prickly pear cactus fibre (nopal) on small intestinal water content and colonic volume demonstrated using magnetic resonance imaging (MRI). Neurogastroenterology and Motility 2018;30:A230.

(60) Stephen AM, Cummings JH. Water-holding by dietary fibre in vitro and its relationship to faecal output in man. Gut 1979; 20:722-729.

(61) Major G, Murray K, Singh G, Nowak A, Hoad CL, Marciani L et al. Demonstration of differences in colonic volumes, transit, chyme consistency, and response to psyllium between healthy and constipated subjects using magnetic resonance imaging. Neurogastroenterol Motil 2018;e13400.

(62) Thomson TJ, Runcie J, Khan A. The effect of diet on ileostomy function. Gut 1970; $11(6): 482-485$.

(63) Wilkinson-Smith V, Major G, Ashleigh L, Murray K, Hoad C, Marciani L et al. In vivo assessment of foods that stimulate intestinal secretions using magnetic resonance imaging: Implications for dietary advice in ileostomy care. Neurogastroenterol Mot 2017; 29:40.

(64) Kon R, Ikarashi N, Nagoya C, Takayama T, Kusunoki Y, Ishii M et al. Rheinanthrone, a metabolite of sennoside $A$, triggers macrophage activation to decrease aquaporin- 3 expression in the colon, causing the laxative effect of rhubarb extract. J Ethnopharmacol 2014; 152(1):190-200. 
(65) Wilkinson-Smith VC, Major G, Ashleigh L, Murray K, Hoad CL, Marciani L et al. Insights Into the Different Effects of Food on Intestinal Secretion Using Magnetic Resonance Imaging. JPEN J Parenter Enteral Nutr 2018.

(66) Lam C, Chaddock G, Marciani LL, Costigan C, Cox E, Hoad C et al. Distinct Abnormalities of Small Bowel and Regional Colonic Volumes in Subtypes of Irritable Bowel Syndrome Revealed by MRI. Am J Gastroenterol 2016.

(67) Cann PA, Read NW, Cammack J, Childs H, Holden S, Kashman R et al. Psychological stress and the passage of a standard meal through the stomach and small intestine in man. Gut 1983; 24(3):236-240.

(68) Larauche M, Kiank C, Tache Y. Corticotropin releasing factor signaling in colon and ileum: regulation by stress and pathophysiological implications. J Physiol Pharmacol 2009; 60 Suppl 7:33-46.

(69) Pritchard SE, Garsed KC, Hoad CL, Lingaya M, Banwait R, Thongborisute W et al. Effect of experimental stress on the small bowel and colon in healthy humans. Neurogastroenterol Motil 2015.

(70) Murray KA, Lam C, Rehman S, Marciani L, Costigan C, Hoad CL et al. Corticotropin-releasing factor increases ascending colon volume after a fructose test meal in healthy humans: a randomized controlled trial. Am J Clin Nutr 2016; 103(5):1318-1326.

(71) Lam C, Chaddock G, Marciani L, Costigan C, Paul J, Cox E et al. Colonic response to laxative ingestion as assessed by MRI differs in constipated irritable bowel syndrome compared to functional constipation. Neurogastroenterol Motil 2016.

(72) Garsed K, Chernova J, Hastings M, Lam C, Marciani L, Singh G et al. A randomised trial of ondansetron for the treatment of irritable bowel syndrome with diarrhoea. Gut 2014; 63(10):1617-1625.

(73) Placidi E, Marciani L, Hoad CL, Napolitano A, Garsed KC, Pritchard SE et al. The effects of loperamide, or loperamide plus simethicone, on the distribution of gut water as assessed by MRI in a mannitol model of secretory diarrhoea. Aliment Pharmacol Ther 2012; 36(1):64-73.

(74) Menys A, Atkinson D, Odille F, Ahmed A, Novelli M, Rodriguez-Justo M et al. Quantified terminal ileal motility during MR enterography as a potential biomarker of Crohn's disease activity: a preliminary study. Eur Radiol 2012; 22(11):24942501.

(75) Menys A, Taylor SA, Emmanuel A, Ahmed A, Plumb AA, Odille F et al. Global small bowel motility: assessment with dynamic MR imaging. Radiology 2013; 269(2):443-450.

(76) Menys A, Butt S, Emmanuel A, Plumb AA, Fikree A, Knowles C et al. Comparative quantitative assessment of global small bowel motility using magnetic resonance imaging in chronic intestinal pseudo-obstruction and healthy controls. Neurogastroenterol Motil 2016; 28(3):376-383.

(77) Khalaf A, Nowak A, Menys A, Marciani L, Taylor SA, Spiller RC et al. Cine MRI assessment of motility in the unprepared small bowel in the fasting and fed state: Beyond the breath-hold. Neurogastroenterol Motil 2019; 31(1):e13466. 
(78) de Jonge CS, Menys A, van Rijn KL, Bredenoord AJ, Nederveen AJ, Stoker J. Detecting the effects of a standardized meal challenge on small bowel motility with MRI in prepared and unprepared bowel. Neurogastroenterol Motil 2019; 31(2):e13506. 